\title{
ChemComm
}

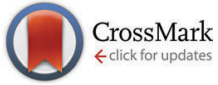

Cite this: Chem. Commun., 2015, 51,8600

Received 6th March 2015

Accepted 14th April 2015

DOI: $10.1039 / c 5 c c 01914 a$

www.rsc.org/chemcomm

\section{Photocatalytic multiphase micro-droplet reactors based on complex coacervation $\dagger$}

\author{
Kangle Lv, ${ }^{a}$ Adam W. Perriman* ${ }^{\mathrm{bc}}$ and Stephen Mann ${ }^{\star c}$
}

\begin{abstract}
We describe the synthesis and characterisation of novel photocatalytic multiphase micro-droplet reactors comprising $\mathrm{TiO}_{2}$ nanosheets dispersed in poly(diallyldimethylammonium) chloride and adenosine 5'-triphosphate or poly(ethylene glycol) 4-nonylphenyl 3-sulfopropyl complex coacervates. We demonstrate significant variations in the degree of equilibrium partitioning of small molecule dyes into the coacervate droplet systems and exploit this behaviour to successfully conduct selective photocatalytic dye degradation.
\end{abstract}

Coacervate micro-droplets are formed spontaneously by liquidliquid phase separation from mixtures of oppositely charged polyelectrolytes in aqueous solution. ${ }^{1}$ Recent studies have demonstrated that complex coacervates can also be prepared using highly charged small molecules such as adenosine triphosphate (ATP) in combination with cationic peptides, polypeptides or polymers to produce molecularly crowded membrane-free protocells. ${ }^{2-4}$ The droplets are typically tens or hundreds of micrometres in size, highly enriched in peptides and nucleotides, stable across a large $\mathrm{pH}$ and temperature range, and capable of sequestering a wide range of low and high molecular weight components including enzymes and their substrates. As a consequence, increased reaction rates and yields have been reported for enzyme cascade reactions confined within the coacervate phase, ${ }^{2,5}$ suggesting that coacervate droplets present an attractive option as multiphase micro-droplet reactors.

In this paper, we explore the possibility of using coacervate micro-droplets for the design and construction of new waterdispersible heterogeneous photocatalytic chemical microsystems. We demonstrate that preformed titania nanosheets $\left(\mathrm{TiO}_{2} \mathrm{NS}\right)$ can be spontaneously sequestered at high concentrations within the chemically enriched coacervate micro-droplets. The $\mathrm{TiO}_{2} \mathrm{NS}$ are

\footnotetext{
${ }^{a}$ Key Laboratory of Catalysis and Materials Science of the State Ethnic Affairs Commission \& Ministry of Education, South-Central University for Nationalities, Wuhan 430074, China. E-mail: lvkangle@mail.scuec.edu.cn

${ }^{b}$ School of Cellular and Molecular Medicine, University of Bristol, BS8 1TD, UK. E-mail: chawp@bristol.ac.uk

${ }^{c}$ Centre for Organized Matter Chemistry and Centre for Protolife Research, School of Chemistry, University of Bristol, BS8 1TS, UK. E-mail: s.mann@bristol.ac.uk

$\dagger$ Electronic supplementary information (ESI) available. See DOI: 10.1039/c5cc01914a
}

prepared by HF etching, ${ }^{6,7}$ and display predominately catalytically active $\{001\}$ facets, ${ }^{8,9}$ which increases the quantum efficiency of these photo-resistant, photo-oxidative nanomaterials. We show that a range of organic dye molecules can be sequestered by the coacervate micro-droplets, and demonstrate that charge or hydrophobic complementarity is responsible for the observed differences in partitioning. Moreover, we exploit these differences to perform selective photocatalytic dye degradation within coacervate droplets containing a mixture of organic dyes.

Positively charged $\mathrm{TiO}_{2} \mathrm{NS}$ (zeta potential $=+26 \mathrm{mV}$ ) were synthesized as described previously (ESI, $\dagger$ Methods). ${ }^{7}$ Aqueous turbid dispersions of photocatalytically active coacervate microdroplets were prepared at $\mathrm{pH} 7-8$ by mixing aqueous solutions of ATP or the anionic polymer surfactant, poly(ethylene glycol) 4-nonylphenyl 3-sulfopropyl ether (KPSE, Fig. S1, ESI $\dagger$ ) with a poly(diallyldimethylammonium) chloride (PDDA, $M_{\mathrm{w}} 100-200 \mathrm{kDa}$, Fig. S1, ESI $\dagger$ ) solution containing a suspension of $\mathrm{TiO}_{2} \mathrm{NS}$. Optical microscopy images showed discrete spherical micro-droplets that were $c a .50$ and $5 \mu \mathrm{m}$ in diameter for the $\mathrm{TiO}_{2} \mathrm{NS} / \mathrm{PDDA} / \mathrm{ATP}$ and $\mathrm{TiO}_{2} \mathrm{NS} / \mathrm{PDDA} / \mathrm{KPSE}$ coacervates, respectively, and which in both cases contained high-contrast material that was attributed to the presence of sequestered $\mathrm{TiO}_{2} \mathrm{NS}$ aggregates (Fig. 1). UV-vis spectroscopic analysis at $325 \mathrm{~nm}$ of the centrifuged coacervate phase and aqueous supernatant gave equilibrium partition coefficients $\left(K=\left[\mathrm{TiO}_{2} \mathrm{NS}\right]_{\text {in }} /\left[\mathrm{TiO}_{2} \mathrm{NS}\right]_{\text {out }}\right)$ of 6150 and 60 for the PDDA/ATP and PDDA/KPSE systems, respectively, consistent with high levels of uptake of the inorganic nanosheets in the molecularly crowded coacervate phases. We attributed the lower $K$ value associated with the $\mathrm{TiO}_{2} \mathrm{NS} / \mathrm{PDDA} / \mathrm{KPSE}$ system to the reduced polarity of the polymer surfactant compared with the ATP constituent.

To assess the potential of the $\mathrm{TiO}_{2} \mathrm{NS}$-containing complex coacervates as photocatalytic multiphase micro-droplet reactors, we first determined whether water-soluble organic molecules could be spontaneously sequestered into the membrane-free micro-compartments. A range of dyes, including Methylene Blue (MB, cationic), Rhodamine B (RhB, zwitterionic), Brilliant Red X-3B (X3B, anionic), and Sulforhodamine B (SRhB, net negatively 


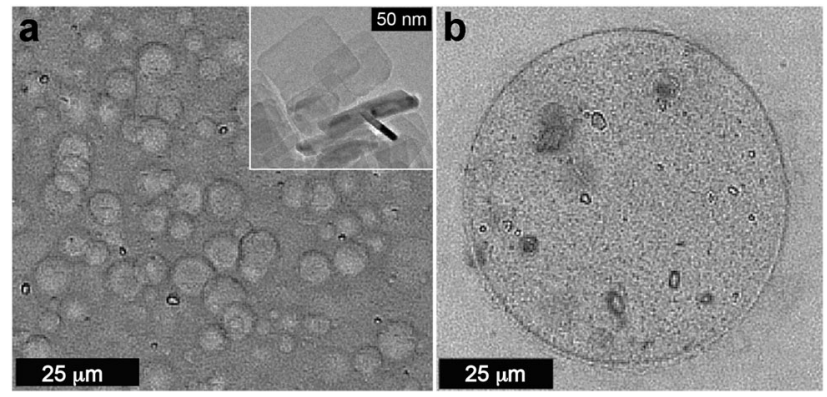

Fig. 1 Optical microscopy images showing (a) population of $\mathrm{TiO}_{2} \mathrm{NS} /$ PDDA/KPSE coacervate micro-droplets, and (b) single $\mathrm{TiO}_{2} \mathrm{NS} / \mathrm{PDDA} /$ ATP droplet. Inset in (a) shows TEM image of the plate-like $\mathrm{TiO}_{2} \mathrm{NS}$.

charged) (ESI, $\dagger$ Fig. S2), were added to the coacervate suspensions at $\mathrm{pH} 7-8$ and the $K$ values determined. Significantly, in all but one case (RhB in PDDA/ATP), the dye molecules strongly partitioned into the molecularly crowded coacervate phases, but the uptake selectivity differed considerably between the PDDA/ ATP and PDDA/KPSE systems. In the former, the following order of $K$ values was observed; X-3B (234) > SRhB (35) > MB (28) > $\mathrm{RhB}$ (0.55), whilst partitioning in the PDDA/KPSE coacervate followed the sequence; $\mathrm{RhB}(38)>\mathrm{SRhB}(24)>\mathrm{X}-3 \mathrm{~B}(12)>\mathrm{MB}$ (6). These differences were primarily attributed to charge or hydrophobic matching between the host and guest components. In this regard, simulations of the $\mathrm{pH}$-dependent $\log D$ partition coefficients ${ }^{10}$ for each dye molecule over a $\mathrm{pH}$ range of 1 to 12 gave constant values between $\mathrm{pH} 7$ and 8 for all the dyes (ESI, $\dagger$ Methods and Fig. S3), and showed decreasing hydrophobic character in the order of $\mathrm{RhB}>\mathrm{MB}>\mathrm{SRhB} \geq \mathrm{X}-3 \mathrm{~B}$. This sequence was inversely correlated to the values of $K$ for the PDDA/ATP coacervates, indicating that polar and charge interactions were the main driving force for sequestration in this system at pH 7-8. Significantly, increased values of $K$ correlated with those dye molecules containing the highest number of negative charges (X-3B and SRhB) (ESI, $\uparrow$ Table S2), suggesting that high levels of sequestration were facilitated by partial displacement of ATP anions associated with electrostatic binding of the guest molecules to PDDA. In contrast, higher $K$ values in the PDDA/KPSE coacervates were associated with the more hydrophobic dye molecules.

Given that a range of dye molecules could be successfully taken up by the complex coacervates, we exploited these host-guest ensembles as membrane-free multiphase micro-droplet reactors. The photocatalytic activity of $\mathrm{TiO}_{2} \mathrm{NS} / \mathrm{PDDA} / \mathrm{ATP}$ and $\mathrm{TiO}_{2} \mathrm{NS} /$ PDDA/KPSE micro-droplets was assessed by exposing suspensions of the coacervates and sequestered dye molecules to UV radiation and comparing the rates of dye molecule degradation to control experiments involving dispersions of $\mathrm{TiO}_{2} \mathrm{NS}$ in water, or coacervate micro-droplets without $\mathrm{TiO}_{2} \mathrm{NS}$. All samples containing $\mathrm{TiO}_{2} \mathrm{NS}$ showed an exponential decay in dye concentration over periods of between 10 and 30 min (Fig. 2 and Fig. S4 and S5, ESI $\dagger$ ), but the decay constants varied considerably (ESI, $\uparrow$ Table S1). For example, the decay constants $(\lambda)$ determined for the photoinduced degradation of $\mathrm{MB}$ in $\mathrm{TiO}_{2} \mathrm{NS} /$ water, $\mathrm{TiO}_{2} \mathrm{NS} / \mathrm{PDDA} / \mathrm{KPSE}$ or $\mathrm{TiO}_{2} \mathrm{NS} / \mathrm{PDDA} / \mathrm{ATP}$ were $0.5,0.1$ and $0.03 \mathrm{~min}^{-1}$ respectively. Interestingly, photocatalysis
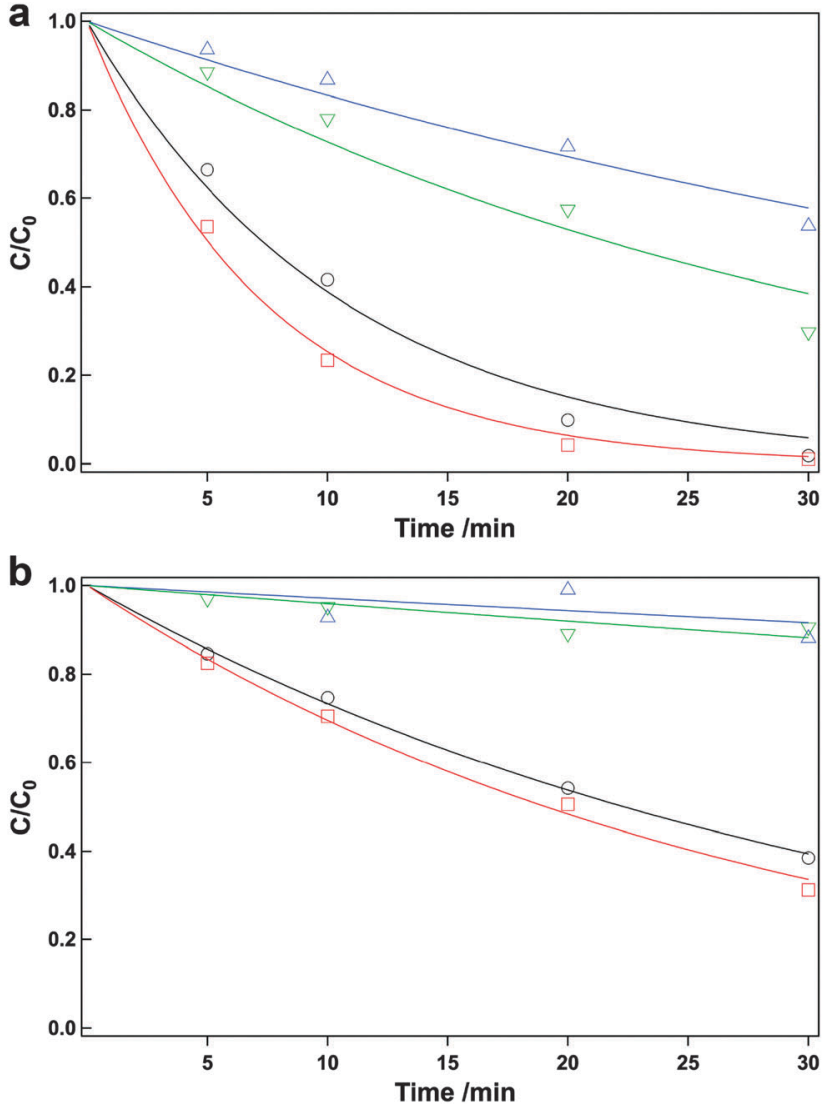

Fig. 2 Photocatalytically induced dye degradation in coacervate microdroplets. Plots show the time-dependent reduction in the normalized dye concentration sequestered into (a) $\mathrm{TiO}_{2} \mathrm{NS} / \mathrm{PDDA} / \mathrm{KPSE}$ and (b) $\mathrm{TiO}_{2} \mathrm{NS} /$ PDDA/ATP; for MB (black line and circles), MB after the addition of $5 \mathrm{M}$ $\mathrm{NaCl}$ (red line and squares), SRhB (blue line and triangles) and SRhB after the addition of $5 \mathrm{M} \mathrm{NaCl}$ (green line and inverted triangles).

in the $\mathrm{TiO}_{2} \mathrm{NS} / \mathrm{PDDA} / \mathrm{KPSE}$ or $\mathrm{TiO}_{2} \mathrm{NS} / \mathrm{PDDA} / \mathrm{ATP}$ micro-droplets was reduced compared with analogous reactions undertaken in the presence of $\mathrm{TiO}_{2} \mathrm{NS}$ dispersed in water. We attributed this to a reduction in UV intensity due to increased light scattering in the droplet phases, or an aggregation-induced reduction of the accessible surface area of the $\mathrm{TiO}_{2} \mathrm{NS}$ catalyst within the coacervate matrix, or both. For example, destabilization of the $\mathrm{TiO}_{2} \mathrm{NS} /$ PDDA/KPSE droplets by addition of aqueous $\mathrm{NaCl}$ such that the turbid dispersions became clear solutions was associated with an increase in the $\lambda$ values for all the dyes (Fig. 2, ESI, $\uparrow$ Table S1 and Fig. S4 and S5). Similarly, the catalytic efficiency values were higher in the $\mathrm{TiO}_{2} \mathrm{NS} / \mathrm{PDDA} / \mathrm{KPSE}$ micro-droplets compared with the bulk coacervate over all $\mathrm{TiO}_{2} \mathrm{NS}$ concentrations studied (ESI, $\dagger$ Fig. S6 and Table S3), confirming that photocatalysis was sensitive to optical transparency. In contrast, minimal changes in $\lambda$ were observed when the $\mathrm{TiO}_{2} \mathrm{NS} / \mathrm{PDDA} /$ ATP coacervates were disassembled (ESI, $\dagger$ Table S1), suggesting that interactions between the components of the coacervate droplet and sequestered $\mathrm{TiO}_{2} \mathrm{NS}$, rather than a loss of incident light intensity due to scattering effects, were responsible for the reduced photocatalytic efficiency in this system. This was consistent with DLS results, which that showed that extensive 
aggregation of $\mathrm{TiO}_{2} \mathrm{NS}$ occurred in the presence of ATP (ESI, $\dagger$ Table S4).

In general, $\mathrm{TiO}_{2} \mathrm{NS} / \mathrm{PDDA} / \mathrm{KPSE}$ micro-droplets were more photocatalytically active than their $\mathrm{TiO}_{2} \mathrm{NS} / \mathrm{PDDA} / \mathrm{ATP}$ counterparts, suggesting that the presence of ATP inhibited dye photodegradation possibly by passivation of the charged $\{00-1\}$ surface of the nanosheets via phosphate-mediated ATP-Ti(Iv) interactions, ${ }^{11}$ or shielding of the dye molecules via $\pi-\pi$ interactions with the adenine group of ATP, ${ }^{4,12}$ or both. The latter appeared to be prominent for SRhB, which did not undergo any significant degradation in the $\mathrm{TiO}_{2} \mathrm{NS} / \mathrm{PDDA} / \mathrm{ATP}$ micro-droplets, or when these droplets were disassembled in the presence of $\mathrm{NaCl}$ (Fig. 2b), or in control experiments involving irradiated samples of $\mathrm{TiO}_{2} \mathrm{NS}$ dispersed in an aqueous solution of ATP (ESI, $\uparrow$ Fig. S7).

The ability to selectively sequester and degrade different dyes using the $\mathrm{TiO}_{2} \mathrm{NS} / \mathrm{PDDA} / \mathrm{ATP}$ micro-droplets was exploited by performing simultaneous dye degradation experiments on MB and RhB with $K$ values of 28 and 0.55 respectively. The dyes were co-sequestered into the coacervate droplets and exposed to UV light. Simultaneous photo-degradation was monitored by changes in the visible excitation absorption bands at $668 \mathrm{~nm}$ (MB) and $555 \mathrm{~nm}(\mathrm{RhB})$, and the exponential decay constants compared with values obtained for mixtures of the dyes in aqueous $\mathrm{TiO}_{2} \mathrm{NS}$ dispersions with or without PDDA present. Both of the aqueous controls exhibited time-dependent reductions in the 668 and $555 \mathrm{~nm}$ absorption bands, signifying simultaneous degradation of MB and RhB (ESI, $\dagger$ Fig. S8 and S9) with associated $\lambda_{\mathrm{MB}} / \lambda_{\mathrm{RhB}}$ ratios of $2.3 \pm 0.2$ (without PPDA) and $0.6 \pm 0.1$ (with PDDA), which were in reasonable agreement with the same ratio $(1.5 \pm 0.1)$ obtained from the individual aqueous dye degradation experiments (ESI, $\dagger$ Table S1). In contrast, analogous experiments performed over two hours using the $\mathrm{TiO}_{2} \mathrm{NS} / \mathrm{PDDA} / \mathrm{ATP}$ microdroplet dispersion showed only a small reduction in intensity of the $555 \mathrm{~nm}$ absorption band along with almost complete loss of the $668 \mathrm{~nm}$ absorption feature to give a $\lambda_{\mathrm{MB}} / \lambda_{\mathrm{RhB}}$ ratio of $18 \pm 4$ (Fig. 3). Thus, it was possible to achieve selective degradation of $\mathrm{MB}$ in the presence of $\mathrm{RhB}$ by spatial confinement of the dye molecules specifically within the $\mathrm{TiO}_{2} \mathrm{NS} / \mathrm{PDDA} / \mathrm{ATP}$ micro-droplets.

In conclusion, this study has shown that photocatalytically active titania nanosheets can be sequestered at high concentrations into coacervate droplets, and used as multiphase micro-droplet reactors. The nanoparticle-containing droplets spontaneously sequester a range of organic dyes with various equilibrium partition constants that are dependent on the chemical functionalities of the guest molecules. We demonstrate photocatalytic dye degradation specifically within the micro-droplets, and utilise differences in partitioning to perform selective photocatalytic degradation in a binary dye system. Our results suggest that the rational integration of catalytic nanoscale objects within membrane-free compartments capable of selective small molecule uptake and storage can be used as a modular approach to the development of hybrid biphasic nano/micromaterials with tuneable properties and reactivity that could have diverse applications in detoxification and bioremediation processes.
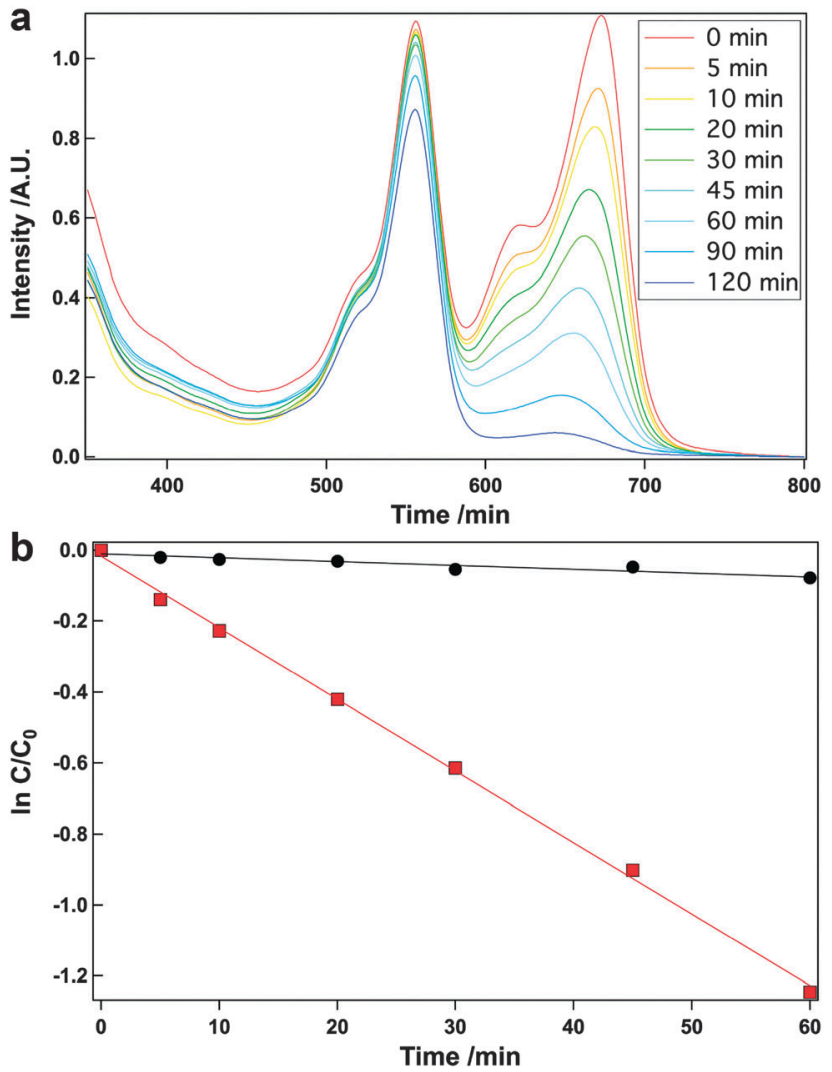

Fig. 3 Selective dye degradation in photocatalytic $\mathrm{TiO}_{2} \mathrm{NS} / \mathrm{PDDA} / \mathrm{ATP}$ coacervate micro-droplets. (a) UV-vis spectra showing time-dependent changes in the intensity of the spectral features from Methylene Blue (MB; $\left.\mathrm{abs}_{\max }=668 \mathrm{~nm}\right)$ and Rhodamine $\mathrm{B}\left(\mathrm{RhB} ; \mathrm{abs}_{\max }=555 \mathrm{~nm}\right)$ co-sequestered within $\mathrm{TiO}_{2} \mathrm{NS} / \mathrm{PDDA} / \mathrm{ATP}$ coacervate micro-droplets. (b) Plots of timedependent changes in the natural logarithm of the normalized peak intensities ( $\ln C / C_{0}$ ) corresponding to MB (red squares) and RhB (black circles). The solid lines are the resulting fits to the data using linear regression.

We gratefully acknowledge the EPSRC (Early Career Fellowship EP/K026720/1), ERC (Advanced Grant) and the National Natural Science Foundation of China (21373275).

\section{Notes and references}

1 A. Prokop, D. Hunkeler, S. DiMari, M. A. Haralson, T. G. Wang, Microencapsulation - Microgels - Iniferters, 1998, vol. 136, p. 1.

2 S. Koga, D. S. Williams, A. W. Perriman and S. Mann, Nat. Chem., 2011, 3, 720 .

3 D. S. Williams, S. Koga, C. R. C. Hak, A. Majrekar, A. J. Patil, A. W. Perriman and S. Mann, Soft Matter, 2012, 8, 6004.

4 T. Y. D. Tang, C. R. C. Hak, A. J. Thompson, M. K. Kuimova, D. S. Williams, A. W. Perriman and S. Mann, Nat. Chem., 2014, 6, 527.

5 J. Crosby, T. Treadwell, M. Hammerton, K. Vasilakis, M. P. Crump, D. S. Williams and S. Mann, Chem. Commun., 2012, 48, 11832.

6 G. Liu, C. H. Sun, H. G. Yang, S. C. Smith, L. Z. Wang, G. Q. Lu and H. M. Cheng, Chem. Commun., 2010, 46, 755.

7 Z. Y. Wang, K. L. Lv, G. H. Wang, K. J. Deng and D. G. Tang, Appl. Catal., B, 2010, 100, 378.

8 H. G. Yang, C. H. Sun, S. Z. Qiao, J. Zou, G. Liu, S. C. Smith, H. M. Cheng and G. Q. Lu, Nature, 2008, 453, 638.

9 H. G. Yang, G. Liu, S. Z. Qiao, C. H. Sun, Y. G. Jin, S. C. Smith, J. Zou, H. M. Cheng and G. Q. Lu, J. Am. Chem. Soc., 2009, 131, 4078.

10 V. N. Viswanadhan, A. K. Ghose, G. R. Revankar and R. K. Robins, J. Chem. Inf. Comput. Sci., 1989, 29, 163.

11 K. L. Lv and Y. M. Xu, J. Phys. Chem. B, 2006, 110, 6204.

12 J. L. Tang, C. Y. Li, Y. F. Li and C. X. Zou, Chem. Commun., 2014, 50, 15411. 